Zeitschriftenartikel:

Begutachtet

Redaktion und Begutachtung

Nele Heise (ID)

Digital Media \& Communication

Researcher, Hamburg

Nils Zurawski (D)

Universität Hamburg

Erhalten: 01. Dezember 2019

Akzeptiert: 29. September 2020

Publiziert: 15. Dezember 2020

Lizenz:

(c) Moritz Klenk

Dieses Werk steht unter einer Lizenz

Creative-Commons-Namensnennung 4.0

(CC-BY 4.0) International

(C) (i)

Datenverfügbarkeit:

Alle relevanten Daten befinden sich innerhalb der Veröffentlichung.

Interessenskonfliktstatement:

Die Autor:innen erklären, dass ihre Forschung ohne kommerzielle oder finanzielle Beziehungen durchgeführt wurde, die als potentielle

Interessenskonflikte ausgelegt werden können.

Empfohlene Zitierung:

Klenk, M. (2020). Stimme, Sprechen, Hören: Von der sinnlich-sinnhaften Verfertigung wissenschaftlicher Erkenntnis beim Sprechen und Hören. kommunikation@gesellschaft, 21(2). https://doi.org/10.15460/kommges. 2020.21.2.623

\section{Stimme, Sprechen, Hören}

\section{Von der sinnlich-sinnhaften Verfertigung wissenschaftlicher Erkenntnis beim Sprechen und Hören}

\author{
Moritz Klenk ${ }^{a^{*}(1)}$ \\ ${ }^{a}$ Hochschule Mannheim \\ * Korrespondenz: klenk@hs-mannheim.de
}

\begin{abstract}
Über die Bedeutung von Podcasts für die Wissenschaftskommunikation für ein breites Publikum hinaus stellt sich unter aktuellen medienevolutionären Bedingungen neu die Frage nach der Bedeutung von Stimme, Sprechen und Hören für die sinnlich-sinnhafte Genese wissenschaftlicher Erkenntnis und Wahrheit. In der dialektischen Entfaltung von Stimme, Hören und Sprechen nach der Schrift zeigt der Beitrag die Notwendigkeit und Möglichkeiten der erkenntnistheoretischen Reflexion von Darstellung und Durchführung von Wissenschaft im Medium Podcasting als Medium von Stimme, Sprechen und Hören. Anhand der Ansätze von Recording Culture (Makagon \& Neumann, 2009), dem Format der Audio Papers (Groth \& Samson, 2016) und dem Sprechenden Denken (Klenk, 2020) werden die Bedeutung der Sinne, des Körpers, des Sprechens und Hörens, der Praxis der Entwicklung sowie des Gesprächs und Selbstgesprächs für die Wissen- und Wahrheitsgenese skizziert.
\end{abstract}

Schlagworte: Sprache, Stimme, Hören, Ästhetik, Sinne, Erkenntnis, Sprechendes Denken, Podcasting, Wissenschaftstheorie 


\section{Einleitung}

Die Wissenschaftskommunikation hat seit einigen Jahren das Medium Podcasting für sich entdeckt. Dabei werden, den Annäherungen an andere digitale Medien ähnlich, vor allem Podcasts als Instrument der ,public science' verstanden und genutzt. ${ }^{1}$ Die für Außenstehende als , hermetisch' erscheinenden fachdisziplinären Forschungen und Theoriediskurse können hier für ein breiteres, fachfremdes wissenschaftliches bis nicht-wissenschaftliches Publikum zugänglich gemacht werden. Auch der vorliegende CfP kennt vor allem diese Potenziale. Erzählende Formate, Interviews, aber auch gebaute Features können hier als bevorzugte Formate erwähnt werden. Podcasts dienen hier vor allem als Medium der Wissensvermittlung. Es schließt sich gerade bei der Prominenz einiger Formate dann aber die weitergehende Frage an: Was sind die Potenziale von Podcasts in der Wissensgenese, in der methodischen und theoretischen Produktion wissenschaftlicher Erkenntnis selbst?

In der gegenwärtigen (medien-)wissenschaftlichen und journalistischen Beschäftigung werden Podcasts meist durchgehend als ein besonders interessantes, neues mediales Produkt; sei es als ein journalistisches, ein unterhaltendes, informatives oder sogar wissenschaftlich fundiertes Produkt und damit Erzeugnis und Ergebnis. Die Praxis der Herstellung, Podcasting als Handeln verstanden, steht hier ganz im Dienst dieses neuen Produkts, bleibt meist unsichtbar oder wird in ihrer Bedeutung im Hinblick auf das Ergebnis verstanden, befragt, analysiert, wenn überhaupt erwähnt. Wenn man von Podcasting spricht, so spricht man meist nur von Podcasts.

Für die Bedeutung von Podcasting für die Wissenschaft ist diese Präferenz nun noch einmal verstärkt; die Produktion bleibt praktisch völlig ohne Bedeutung für die Reflexion. Damit ist nun nicht gemeint, dass nicht auch allfällig ein Lob der Einfachheit des Podcastens gegenüber anderen medialen Produktionsweisen fällt oder es keine Ratgeber gäbe, wie man einen Podcast ,mit einfachen Mitteln' selbst machen könnte; unter Bedingungen von Corona sind solche Anleitungen erst jüngst wie Pilze aus dem Boden geschossen und auch die Vorteile des Podcasting finden immer häufiger in verschiedenen Kontexten der Wissensvermittlung, Hochschuldidaktik oder zur Aufbereitung von Konferenzen Erwähnung.

Aber: Was schon für das Schreiben wissenschaftlicher Texte eine kleine Ewigkeit brauchte, nämlich dieses als eine Praxis eigener Materialität und Performativität zu verstehen und darin in seiner Bedeutung für die Wissensproduktion zu reflektieren (so prominent im Zuge der ,Writing Culture Debatte', vgl. Clifford, 1986a; Geertz, 1987; Marcus, 2007; Marcus \& Cushman, 1982), ist für das Podcasting noch lange nicht in Sicht- oder Hörweite.

Wenn ich heute vom Sprechen und Hören schreibe, dann sind damit sofort diese beiden Dimensionen der Praxis wie des ,Produkts' aufgerufen. Genauer ver-

1 Vergleiche etwa die umfangreiche Sammlung von der Wissens- und Wissenschaftspodcasts auf https: //wissenschaftspodcasts.de/podcasts/ (Zugriff am 10.09.2020). Die allermeisten hier geführten Podcasts richten sich an ein breiteres Publikum oder zumindest deutlich über enge fachdisziplinäre Diskursgrenzen hinaus. 
suche ich mit diesem Beitrag dem Ungleichgewicht entgegenzuwirken, indem ich beide Seiten als Praxen verstehe, als Praxis des Sprechens und Praxis des Hörens, und zugleich diese Unterscheidung infrage zu stellen versuche. Dies betrifft dann jedoch die Ebene des oben formulierten blinden Flecks der wissenschaftlichen Reflexion von Podcasting in seiner Bedeutung für die Wissenschaft: als Produkt und dann vorrangig als Produkt der Wissensvermittlung bleibt die Bedeutung des Sprechens und Hörens als sinnlich-ästhetische Praxis sowohl der Rezeption, aber vor allem auch der Verfertigung unbeachtet. Die Frage meines Beitrags lässt sich also wie folgt formulieren: Wie und in welchen Formen sind Wissensgenese und -vermittlung als sinnlich-ästhetische wie sprachlich-begriffliche Praxen in Stimme, Sprechen und Hören für wissenschaftliches Podcasting zu reflektieren?

\section{Stimme}

Wenn von Wissenschaft die Rede ist, so geht es fast ausschließlich um Schrift und geschriebenen Text. Die dominante visuelle Kultur findet in der schriftlichen Form der Darstellung wissenschaftlichen Wissens ihre Entsprechung; ${ }^{2}$ das Sprechen und Hören der Sprache bleibt stumm. Was zählt, ist oft nur der Text. Einige der vielen Gründe, die hierfür auch explizit angeführt werden können, sind etwa die Genauigkeit schriftlicher Notation, die schnellere Vermittlung größerer Mengen an Information, die Einfachheit der (technischen) Vermittlung oder die Entkopplung von mit der Stimme verbundener Subjektivität, die es im Sinne einer positiv(istisch)en Wissenschaftlichkeit zu vermeiden gilt. Ist es nicht einfacher, schneller, effizienter und objektiver, neutraler, sachlicher, sauberer, wissenschaftliche Erkenntnisse zu lesen, statt eine menschliche Stimme zu bemühen?

Die wissenschaftliche Kritik am Medium der Stimme ist dabei sehr unterschiedlich, nicht selten paradox: so steht die Stimme in einem Fall für den Sinn selbst oder die Unvermitteltheit des Ausgesprochenen, im anderen wird ihr mangelnde Distanz und Subjektivität vorgeworfen; sie wird für die (Stimm-) Färbung der Inhalte verantwortlich gemacht. Wird sie im Bereich der Rhetorik verortet, wird ihr eine Überredungsqualität unterstellt, die der Notwendigkeit zur streng wissenschaftlichen Argumentation widerspräche. Die Stimme aber bietet vor allem eine Gefahr, nämlich ihre Unberechenbarkeit oder um es mit Blumenberg zu sagen ihre Unbegrifflichkeit (vgl. Blumenberg, 2007). Die Stimme als Phänomen und Gegenstand ist immer mehr als in je einzelnen begrifflichen Proben erfasst werden kann.

Sprachwissenschaftliche Handbücher, auch wenn man gerade hier eine gewisse Zuständigkeit für das Phänomen vermuten könnte, bieten kaum „das Stichwort Stimme [...]. Der Ort, an dem das verfügbare Wissen auftaucht, sind vielmehr speziellere Unterbegriffe wie Stimmanalyse, Stimmlage, Stimmqualität,

2 Auch wenn hier kein Kausalzusammenhang notwendig aufgemacht werden soll: die schriftliche Darstellung und alle Metaphern der Erkenntnis und Wahrheit sprechen die Sprache des Auges oder Sehsinns. Dass sich dann erst zeigt, als Ausdruck für ,wissenschaftlich erwiesen' sichtbar wird, was aufgeschrieben werden kann und wird, ist kein Zufall. 
Stimmstörungen, Stimmverstellung, Stimmhaftigkeit etc.“ Das zeige, „dass eine genaue Analyse dieses Phänomens nur über die einzelnen Aspekte möglich ist." (König \& Brandt, 2006, S. 111) Möglich oder nicht, dieser Vermutung entsprechen dann auch eine mittlerweile unüberschaubare Zahl der Studien zum Phänomenbereich von Stimme und Stimmlichkeit in ebenfalls zahllosen Bereichen von Kultur und Gesellschaft.

Die sortierende Zerlegung des Phänomens in disziplinär bearbeitbare Teilbereiche überzeugt jedoch nicht. In der Schwierigkeit mit dem Phänomen und Gegenstand der Stimme in seiner ganzen Bedeutung ${ }^{3}$ zeigt sich das ihr eigene Merkmal, dass sie sich eben gerade nicht auf physikalische, auditive, körperliche, psychische, soziale oder andere Perspektiven begrenzen lässt. Die Stimme transportiert immer schon ein Mehr. Mladen Dolar formuliert etwa in seiner philosophischen Theorie der Stimme:

„Eine Stimme zu hören und eine Stimme von sich zu geben, ist also gleichermaßen ein Exzess, ein Übermaß an Autorität auf der einen Seite und ein Übermaß an Ausgesetztsein auf der anderen. Wegen des direkten, schutzlosen Übergangs ins Innere gibt es ein Zuviel an Stimme, die aus dem Innern stammt - sie bringt mehr, und anderes, an den Tag, als einem lieb ist." (Dolar, 2011)

Im Anschluss an Roland Barthes beschreibt Dieter Mersch diese Qualität der Stimme als einen ihr eigenen „Überschuss“; sie entzieht sich der begrifflichen Bestimmung. „Sie zeigt sich als nichtaufzeichenbarer Rest, als Residuum oder permanente Reserve. Bestenfalls negativ bezeichenbar, inhäreriert ihr die Paradoxie einer Präsenz der Nichtpräsenz." (Mersch, 2006) Die Stimme lässt sich daher zugleich als ein Medium als auch nicht als solches, sondern eigenes Phänomen, die Darstellung und das Eindrücken der Ausdrücke als sprachliche immer Übersteigendes verstehen.

Im Unterschied zur Schrift oder genauer: gedruckter Schrift, birgt die Stimme notwendig immer ein Mehr, einen Überschuss. Sie ist nie nur Lautzeichen oder Medium im Sinne der selbst nicht mitbeobachtbaren Menge lose gekoppelter Elemente, die temporäre Kopplung zu fixen Formen ermöglichen. ${ }^{4}$ Zwar kann auch die Schrift und selbst die fast entkörperlichten Zeichen gedruckter Buchstaben auf ihre ästhetische Qualität, ihre sinnlich-wahrnehmbare Gestalt und Gestaltung, auf Typographie, Form und vor allem in ihrer Fülle an Differenzierungen vergleichend beobachtet werden, doch erfordert dies einen Wechsel der Perspektive (oder der Unterscheidung). Es muss bewusst eine neue Unterscheidung eingeführt werden, gegen das Medium als Medium. Die Stimme dagegen trägt diese als ihre Körperlichkeit notwendig mit. Sie eignet sich damit immer nur auch zum Ausdruck von Sprache und der Vermittlung von sprachlich verfasstem Sinn; die Grenze des Sinns lässt sich nicht überhören, die ästhetische Dimension ist ihr konstitutiv.

3 Es muss natürlich angemerkt werden, dass sehr wohl ein alltagssprachlicher oder einfacher Begriff der Stimme als etwa physiologisches Phänomen oder Lautorgan des Menschen in reger Verwendung ist, sowohl innerhalb als auch jenseits wissenschaftlicher Diskurse. Dieser ,Begriff', der meist nichts weiter als ein alltagsplausibler Name für eben jenes Mysterium der Stimme ist, ist hier nicht gemeint.

4 Vgl. etwa die Medium/Form-Unterscheidung nach Fritz Heider (Heider, 2005). 
Es ist dieser Zusammenhang, der die Stimme als Medium auszeichnet und zugleich darin als reflexives Medium ihre eigene Medialität mitbeobachtbar macht, indem sie die Grenze immer zugleich mitführt. Die Stimme ist selbstbewusstes Medium. Ein solches Verhältnis ist aber ein dialektisches, ein widersprüchliches und widerständiges. Der Vielzahl der Medienbegriffe zum Trotz lässt sich für einen großen Teil derselben sagen, dass ihnen zufolge eine solche zeitgleiche Mitbeobachtung als das Vermögen zur Formbildung beschränkend oder zu diesem in Widerspruch tretend erachtet werden müsste. Zwar gilt „the Medium is the message" (Mcluhan, 1994, S. 7ff) als Gemeinplatz. Doch selbst wenn in medienwissenschaftlicher Hinsicht die Möglichkeiten zur Formbildung von der Qualität (und das heißt immer: Materialität) des Mediums mitbestimmt sind, so bedarf die Realisierung der Formbildung letztlich des Verschwindens des Mediums. Luft transportiert den Klang nur, solange sie nicht selbst zu rauschen beginnt; es lässt sich nur lesen, insofern von der Schönheit der Linien für den Moment abgesehen werden kann. Die Stimme dagegen fordert genau jene Dialektik heraus, lockt sie hervor, bringt die Darstellung selbst zur Darstellung.

Roland Barthes nennt dies die „Rauheit der Stimme“(Barthes, 2015a). In dem Text aus einer Reihe von Texten zum „Körper der Musik” analysiert Barthes die Qualität der Stimme am Fall der Singstimme. Es geht ihm um den „präzise abgesteckten Raum [...], in dem eine Sprache einer Stimme begegnet." (ebd., S. 270) Zwar verhandelt Barthes hier vordergründig oder auch im Genaueren eine neue Sprache für die Beschreibung von Musik und Klang, doch müssen seine Überlegungen weiter gedacht werden: Die Rauheit der Stimme ist nicht nur eine Qualität der Singstimme, sondern jeder Stimme insofern Stimme im Aufeinandertreffen mit Sprache immer beides hervorbringt: Musik und Sprache. $^{5}$

In einem ganz konkreten Sinn ist die Rauheit der Stimme die Materialität des Körpers, der sich in ihr notwendig einschreibt. Die Stimme trägt die Spur der Körper, mit denen sie untrennbar verbunden ist. Dies gilt sowohl für die Körper der Sprechenden als auch der Hörenden (s. Abschnitt 2). Die ,Rauheit', die Materialität der Stimme sprengt das Sender-Empfänger-Modell der Kommunikation damit in mindestens zweifacher Weise: zum einen ist sie der unabtrennbare, nicht auslöschbare Rest oder das surplus des Körpers in der gesprochenen Sprache. Sie ist Widerspruch und nichtauflösbare Materialität gegen den Sinn der Sprache, welche oft genug zu schnell auf Bedeutungszusammenhänge allein ,bereinigt' wird. Zum anderen ist schon das Phänomen der Stimme immer notwendig intersubjektiv, eine Frage der Resonanz mindestens zweier Körper, deren korporeale, ästhetisch-sinnliche Wahrnehmung konstitutiver Bestandteil des Verstehensprozesses ist.

Wenn ich hier verharmlosend von Verstehensprozessen schreibe, dann darf dies nicht darüber hinwegtäuschen, dass es sich dabei um wissenschaftlich grundlegende, erkenntnistheoretische und -praktische Fragen handelt. An die vermeintlich notwendige Distanz der Schrift, ihrem ungebrochenen

5 An anderer Stelle schreibt Barthes sogar strenger: „Was ist also die Musik? [...] eine sprachliche Qualität.” (Barthes, 2015b, S. 185, Her.i.O.). 
Nimbus der Neutralität gewöhnt, den eher noch wachsenden Hoffnungen und dem sich noch verstärkenden Glauben an die Möglichkeit ,objektiver' Erkenntnis verpflichtet, sind diese Fragen der Stimme fast ausschließlich philosophische, künstlerische oder mindestens marginale Gegenstände wissenschaftlicher Reflexion. ${ }^{6}$ Auch die Konjunktur der Stimme als Gegenstand in den vergangenen 20 Jahren hat daran praktisch kaum etwas geändert. Im Zuge der medialen Entwicklungen des Mediums Podcasting kann allerdings die leise Hoffnung geweckt werden, dass sich daran etwas ändern könnte.

Wer heute von Stimme als Mittel der Erkenntnis schreibt oder spricht, kann selbstverständlich kein bloßes Zurück in vorschriftliche Zeiten meinen. Die Kritik und Reflexion der Stimme, um die es mir hier wie vielen anderen andernorts geht, ist keinesfalls romantische Vorstellung einer Zeit des noch unentfremdeten ,natürlichen' oder gar unmittelbaren Kommunizierens im Sprechen. Es geht nicht um Unmittelbarkeit und nicht um ein Einholen der Gefühle für eine angeblich gefühllos gewordene Wissenschaft. All solche Vermutungen sind blanker Unsinn. Im Gegenteil: Eine Auseinandersetzung mit der Stimme, dem Sprechen und Hören im Kontext wissenschaftlicher Erkenntnisproduktion und dem Ringen um das, was wissenschaftliche Wahrheit genannt werden mag, muss nicht zuletzt die mediale Geschichte der Wissenschaft voraussetzen. Die Stimme, das Sprechen und Hören sind neu zu denken, iterativ, dialektisch, als Aufhebung und Entwicklung und immer wieder Neuentdeckung der Medien der Darstellung und Durchführung von Wissenschaft.

Die Stimme bleibt im Diskurs ungehört, wie auch der Körper, dem die Stimme entspringt, ${ }^{7}$ die meiste Zeit unter der Herrschaft des ,reinen Geistes' und christlich gefärbter Geisteswissenschaft als fleischlich notwendiges Übel missachtet wurde. Bis heute gilt etwa für weite Teile der Soziologie, dass „[d]em Körper [...] die Rolle eines Instruments zugewiesen [wird], über das der Akteur verfügen kann, das gesellschaftlich prägbar, individuell bearbeitbar, grundsätzlich beherrschbar und instrumentell einsetzbar ist." (Weihrich, 2017, S. 33) Wie der Körper als Instrument der Handlung dieser als eigentlicher Gegenstand des Interesses untergeordnet, so sehr er theoretisch wie methodisch vernachlässigt worden war und erst nach vielen Iterationen der cultural turns (vgl. Bachmann-Medick, 2009) heute relevant gesetzt ist, so ist die Stimme noch immer bloßes Instrument der Sprache. Die Sprache wiederum ist ein von all ihrer Färbung und Mehrdeutigkeit möglichst zu befreiendes Medium begrifflicher Exaktheit der Wissenschaft.

Wenn ich im Folgenden nun zum Hören und Sprechen komme, dann nur zu dem Preis eine große Vielzahl so relevanter Fragen zur Stimme als Medium der Wissenschaft ungenannt zu lassen. Etwa die Frage nach der Macht der Sprecher:innenpositionen, Stimme und Gender (vgl. etwa Butler, 1997; Krämer, 2001, S. 241-260), Stimme und Rassismus ${ }^{8}$, oder andere Fragen der Cultural

6 An dieser Stelle mag auch auf linguistische Definitionen der Stimme verwiesen werden als „das, was nichts zur Sinngebung beiträgt" (Dolar, 2014, S. 24, Herv..O.; vgl. im Anschluss an Lacan: Miller, 2007, S. 141).

7 "Was Sprache und Körper gemeinsam haben, ist die Stimme, doch ist die Stimme weder Teil der Sprache noch Teil des Körpers." (Dolar, 2014, S. 100, Herv.i.O.).

8 Hier sei zumindest auf eine hörenswerte Serie des Podcasts The Heart mit dem Titel „Divesting from 
Studies. Diese Auslassungen sind umso unverzeihlicher als sie ganz wesentlich für ein Verständnis der Bedeutung der Stimme als jenes unbegriffliche ,Mehr als ein Medium' sind. Da ich hier über die sinnlich-sinnhafte Verfertigung der Erkenntnis beim Sprechen und Hören schreibe, hoffe ich dennoch auf Nachsicht. Insofern die diskutierten Aspekte zugleich immer über sich hinausweisen, wie schon in der Sache der Stimme selbst veranlagt, können aber bereits daraus politische, ethische und kritisch-engagierte Konsequenzen gezogen werden. Dass es sich bei Fragen der Stimme immer notwendig auch um politische Fragen handelt, liegt nahe (vgl. im Anschluss an Hannah Arendt auch Klenk, 2020, S. 23ff). Bereits als Körperphänomen ist die Stimme notwendig politisch in dem Sinne, dass der Körper des Menschen das letzte Mittel zur minimalen Inklusion darstellt, gerade wenn die ,Person' selbst aus allen sozialen Systemen exkludiert wurde. „Der Körper ist oft das einzig verbliebene Kapital, das die vom ,harten Ausschluss' Betroffenen im Kampf um die knappe Ressource Aufmerksamkeit einzusetzen vermögen." (Schroer, 2004, S. 447). Die Stimme bekommt hier als Schreien ganz ohne Worte und selbst noch als Skandieren besondere Bedeutung; die Philosophin Petra Gehring nennt dies präzise als die „Körperkraft der Sprache“ (vgl. Gehring, 2019, S. 8-32, 121143). Diesen Körper und seine politischen Implikationen gilt es im Folgenden mitzudenken.

\section{Hören}

Wer von der Stimme spricht darf vom Hören nicht schweigen. Auch wenn das vermeintlich passiv-rezeptive Hören in einer aufs (aktive) Handeln hin ausgerichteten Kultur schwach und unscheinbar scheint oder Ausdruck niedrigerer Hierarchieebenen (Befehlsempfänger:innen, Schüler:innen, Gläubige, usw.) ist, ${ }^{9}$ erfährt es im Kontext des Podcastings eine bemerkenswerte Entwicklung.

Für ein Nachdenken über die Bedeutung der Stimme für die Produktion von Erkenntnis ist das Hören von entscheidender Bedeutung. Erst das Hören, ein Vernehmen, genauer ein Hinhören, als Horchen und Zuhören (vgl. auch Barthes, 2015c) komplementiert die Stimme zu jener Form der Darstellung und Durchführung von Erkenntnis in der Dialektik von Materialität, Klang, Sprache und Sinn. Wie die Stimme selbst ist auch das Hören, zumindest in dem über den Hörsinn hinausgehenden Verständnis eine Körpertechnik (mit Mauss, 1989). Es ist klar, dass hier nicht nur von Sprache und Sprechen, oder gar von Argument und Widerrede die Rede sein kann, und das nicht einmal, wenn es im strengen Sinn um wissenschaftliches Sprechen geht (etwa in Podcasts, aber auch in Radioformaten, auf Konferenzen, in Gesprächen oder im Seminar). Das Hören fordert als Körpertechnik und Form sinnlich-ästhetischer Wahrnehmung den Körper ein, der sich nicht auf die Bedeutung, den Sinn der Sprache begrenzen lässt. Und doch ist auch das Hören des Klangs ein Hören von Verweisen: Sprache, Sinn und Klang haben diese Verweisstruktur gemeinsam

people pleasing" von Nicole Kelly (Kelly, 2020) verwiesen.

9 Schon der Ausdruck, jemandem hörig sein' zeigt die eindeutige Präferenz für das Sprechen, genauer ein Sprechen als Befehlen, Unterweisen, Belehren, usw. 
(vgl. Nancy, 2010, S. 17f).

Dieses differenzierte (Zusammen-)Spiel von Verweisen lässt auch methodisch solches Hören als aktive Tätigkeit weiterentwickeln. Der Komponist und Filmtheoretiker Michael Chion hat hier drei ,Modi' des Hörens vorgeschlagen (vgl. Chion, 1994, S. 25-34), die jenes komplexe Spiel der Verweise zu entschlüsseln suchen. Chion unterscheidet kausales Hören (vgl. ebd., S. 25ff.), welches bei Geräusch und Klang deren Ursache und Quelle zu bestimmen sucht; semantisches Hören (vgl. ebd., S. 28f.), welches - besonders in der Sprache - Bedeutung und Sinn zu verstehen ermöglicht; und schließlich reduziertes Hören (vgl. ebd., S. 29ff.), welches die Aufmerksamkeit auf den Klang als solchen richtet - mit erschütternden Folgen für vermeintlich eindeutige Höreindrücke. Das Mehr, der nicht reduzierbare Überschuss der Stimme, von dem oben die Rede war und welcher immer schon das Hören bestimmt, die Sprache affiziert und Semantik zu sprengen vermag, wird durch dieses Hören näher bestimmbar, expliziert aus dem Zusammenspiel herausgelöst und so der Reflexion zugänglich. In diesem Kontext lese ich auch Nancy:

„Ganz Ohr sein, lauschen, das ist immer am Saum des Sinnes sein, oder in einem Rand- und Außensinn, und als wäre der Klang eben nichts anderes als dieser Saum, diese Borte oder dieser Rand - zumindest der musikalisch gehörte Klang." (Nancy, 2010, S. 16)

Jener gemeinsame Raum von Sinn und Klang, der im Hören von Stimmen aufgespannt wird, kann ,ganz allgemein als Raum eines Selbst oder eines Subjekts definiert werden" (ebd., S. 19). Bedenkt man den eingangs erwähnten Vorwurf, mündlich-stimmliche Sprache sei subjektiv, mag dies kaum überraschen. Mit Uwe C. Steiner lässt sich sogar zeigen, „,wie eng eine der revolutionärsten Gedankenfiguren des 18. Jahrhunderts, die Entdeckung der Subjektivität, mit dem Ohrenklang verschwistert ist." (Steiner, 2012, S. 57) Doch geht der Begriff des Subjekts, um den es mir hier im Zusammenhang des Hörens geht, über seine Geburt in der „akustische[n] Introversion“ (ebd., S. 57) hinaus. $^{10}$

Wenn es nicht um das Subjekt im Sinne eines bloß sogenannten ,subjektiven' Fühlens geht, muss es radikaler gedacht werden. Jean-Luc Nancy folgend ist Hören als ein wahrnehmendes Erkennen (aisthesis) im Anschluss an Aristoteles und damit immer ein se-sentir-sentir, ein sich spüren Spüren: „Das Spüren ist Subjekt, oder es spürt nicht." (Nancy, 2010, S. 18) Das Hören des Klangs wirkt in mir, betrifft die Unterscheidung von Innen und Außen (vgl. auch Mühleis 2018). Es schreibt an einem Selbstbewusstsein, da das Hören des Klangs ein Wiederklingen im Innen ist (vgl. Nancy, 2010, S. 18ff). Insofern das Hören der Stimme meinen eigenen Körper mithin ganz wörtlich verstanden im Sinne ,otoakustischer Emissionen ${ }^{11}$ beansprucht, wird die körperliche Wahrnehmung auf sich selbst verwiesen. Im Widerstreit zwischen Rauheit

10 Der Vorwurf der Subjektivität allerdings bezieht sich dennoch auf den hier diskutierten Begriff, wenngleich in positivistischer Verpflichtung eines unkritischen Begriffs der ,Objektivität', der all zu oft Inbegriff von Wissenschaftlichkeit zu sein behauptet (vgl. hierzu die Kritik Adornos weiter unten).

11 Der Begriff otoakustische Emissionen bezeichnet akustische Aussendungen des Innenohrs nach Außen, d.h. entgegen der Richtung des eintreffenden Schalls. In anderen Worten: Geräusche, die das (Innen-)Ohr selbst verursacht. 
und Sinn der Sprache in der Stimme wird das Hören reflexiv.

Zugleich geht das Subjekt hier über jenen Begriff hinaus, greift strenger die Unterscheidung von Subjekt und Objekt als erkenntnistheoretische Dialektik auf. Salomé Voegelin schreibt hierzu:

"Listening cannot contemplate the object/phenomenon heard separate from its audition because the object does not precede listening. Rather, the auditory is generated in the listening practice: in listening I am in sound, there can be no gap between the heard and hearing, I either hear it or I don't, and what I perceive is what I hear. [...] The listener is entwined with the heard. His sense of the world and of himself is constituted in this bond." (Voegelin, 2010, S. 5)

Wie sehr der Vorwurf der Subjektivität der Stimme hier die erkenntnistheoretisch komplexe Situation verkennt, zeigt sich weiter im Anschluss an Adornos Warnung vor der Perversion der Unterscheidung von objektiv und subjektiv [vgl. Adorno (2003a), Aphorismus 43: „Bangemachen gilt nicht“]. Das Subjekt des Hörens kann gerade nicht als transzendentales, philosophisches Abstraktes, sondern muss als konkret-empirisches Subjekt verstanden werden: "It is this particularity of the listening subject in the contingency of his experience that has to be kept in mind, in order, according to Adorno, not to turn the individual subject already into an (objective) universal;" (Voegelin, 2010, S. 14) und weiter: "It is the lived and concrete experience that constitutes the world as a sonic life-world and the subject reciprocally generated within it." (ebd.:, S. 15).

Zugleich geht jenes Hören über das auch psychologisch zu verstehende Subjekt hinaus. Roland Barthes weist darauf hin, dass ,nicht das psychologische ,Subjekt' in mir hört; die Lust, die es sich erhofft, verhilft ihm nicht dazu, sich zu festigen, sich auszudrücken -, sondern, im Gegenteil, zum Selbstverlust.“ (Barthes, 2015a, S. 277)

Was hier als unversöhnlich oder widersprüchlich erscheint, bezeichnet nichts anderes als die Bewegung der Präsenz, die das Hören als Denken bedeutet, die eben keine „Position des Gegenwärtig-Seins“ meint: „Genau das ist sie nicht." (vgl. Nancy, 2010, S. 25ff) Die Position des Subjekts festigt sich und stellt sich zugleich infrage; man hört in der Stimme der Anderen die Rauheit anderer Körper und wird im Wiederklingen des hörenden eigenen Körpers zugleich auf diesen eigenen Körper verwiesen. In beiden Fällen sprengt das Hören das bloße Sinn- und Zeichengeschehen der Sprache als Bedeuten auf. Subjekte sind aufeinander verwiesen; Subjektivität ist im Hören der Stimme in der Interkorporalität auf sich selbst und über sich hinaus verwiesen.

In der Auseinandersetzung mit dem psychoanalytischen Gespräch beschreibt Barthes das Zuhören dann auch in seiner ganzen Dramatik als ein riskantes sich dem Begehren der Anderen Aussetzen:

„Die Anerkennung des Begehrens der anderen kann also keineswegs in der Neutralität, im Wohlwollen oder im Liberalismus entste- 
hen: Dieses Begehren anerkennen bedingt, dass man darin eintritt, hineinschlittert und sich schließlich darin befindet. Das Zuhören existiert nur unter der Bedingung, dass ein Risiko eingegangen wird, und wenn es davon losgelöst werden muss, damit es zur Analyse kommt, so keineswegs mit Hilfe eines theoretischen Schildes." (Barthes, 2015c, S. 260)

Barthes folgend geht es hier schon lange nicht mehr um ein ,Hören an sich' was immer das wäre - sondern vielmehr um ein Hören als historische Körpertechnik; das Hören selbst ist sozial, kulturell und konkret empirisch bestimmt, gestaltet. Das Hören ist immer ein Hören seiner Zeit:

„Zweitens haben die durch den Hörakt bedingten Rollen nicht mehr dieselbe Starrheit wie früher; es gibt nicht mehr auf der einen Seite den Sprechenden, der sich ausliefert und gesteht, und auf der anderen den Zuhörenden, Schweigenden, Urteilenden und Bestrafenden; was nicht besagt, dass der Analytiker zum Beispiel genauso viel spricht wie sein Patient; und zwar deshalb, weil, wie gesagt, sein $\mathrm{Zu}-$ hören aktiv ist, einen Platz im Spiel des Begehrens einnimmt, dessen Schauplatz, die Sprache ist: Man muss es wiederholen, das Zuhören spricht. [...] Man glaubt zur Befreiung des Zuhörens brauche man nur selbst das Wort zu ergreifen - wo doch ein freies Zuhören im Wesentlichen ein Zuhören ist, das zirkuliert, permutiert und durch seine Beweglichkeit das starre Netz der Sprecherrollen auflöst: Eine freie Gesellschaft ist unvorstellbar, wenn man im vorhinein akzeptiert, in ihr die alten Orte des Zuhörens zu erhalten: die des Gläubigen, des Schülers und des Patienten." (Barthes, 2015c, S. 262)

Das Zuhören selbst, als intentionale Form des Hörens spricht. Nicht nur die Stimme im Sprechen zu erheben ist damit von epistemologischer, politischer oder anders transformativer Bedeutung. Im Kontext der epistemologischen Bedeutung der Konstitution von Wissen und Wahrheit im Medium des Podcastings kann dies kaum überschätzt werden.

\section{Sprechen}

Wenn ich nun auf das Sprechen zu schreiben komme, so kann dieses nur im Kontext des bisher Gedachten verstanden werden. Wieder und neu anhebend geht es dabei um die Frage nach seiner Bedeutung für die Verfertigung von Erkenntnis. Man kommt kaum umhin dabei Heinrich von Kleists Überlegungen „,[ü]ber die allmählichen Verfertigung der Gedanken beim Reden“ (von Kleist, 2002) zu erwähnen, auch wenn die dort diskutierten Ideen die Frage nach dem Sprechen nur am Rande betreffen und dafür kaum Erhellendes beitragen; der Fokus eines Gegenüber, mittels dessen man sich selbst zur Explikation der Gedanken ,überredet', ist bestenfalls ein kleiner Teil der relevanten Zusammenhänge des Sprechens. Eher schon gilt es die großen sprachtheoretischen und -philosophischen Positionen zur Frage Gibt es eine Sprache hinter dem Sprechen? 
(vgl. besonders Krämer \& König, 2002; Krämer, 2001) im Gespräch zu behalten. Im Folgenden soll aber das Sprechen vor allem als Praxis verstanden werden, soll es also um die Performanz des Sprechens als Mittel der Erkenntnis gehen.

Das Sprechen von der Stimme her verstehend, muss man genauer auf die Körperlichkeit dieser Praxis achten: die Bewegungen des Mundes, die Vertrautheit der Aussprache, der Worte, die Geschwindigkeit der Zunge, die Artikulation einzelner Buchstaben und Silben, das Hören des Klangs der Stimme im eigenen Körper, und darüber hinaus der ganze Körper in seiner ästhetischsinnlich wahrnehmbaren Materialität, die Schärfe der Zähne, der Klangraum im Mund, Hals, in der Nase, im Bauch. Und nicht zu vergessen die Synästhesie des Zusammenspiels aller Sinne und ihrer Übertragungen: das Hören der Farben des Klangs, der Geschmack der Worte. ${ }^{12}$

Im Anschluss an Giorgio Agamben sagt Alice Lagaay, die Stimme des Sprechens liegt „an der Schwelle zur Bedeutung“ (Lagaay, 2011, S. 113, Herv.i.O.) Es braucht daher eine „negative Philosophie der Stimme“ (ebd., S. 112):

„Für ihn [Agamben] ist wesentlich, dass der Mensch geboren wird, ohne sprechen zu können. Das bedeutet, dass unsere erste Erfahrung von Stimme - neben dem Hören der Stimme der Mutter beziehungsweise Menschen der Umgebung - eine Erfahrung der Stimme ist, die nichts sagt. Sie ist aber auch nicht nur sinnloses Geräusch. Diese Stimme ist in diesem Sinne negativ, weil sie, im Unterschied etwa zur Stimme des Tiers (phoné animale), das von Anfang an im Einklang mit seiner Sprache ist, „nicht mehr nur Geräusch ist, aber auch noch nicht Bedeutung". In dieser Erfahrung der Stimme an der Schwelle zur Bedeutung liegt für Agamben ein Schlüsselerlebnis von Potenzialität". (Lagaay, 2011, S. 113, Herv.i.O.)

Bei der Frage nach der Bedeutung der Stimme für das Denken geht es damit um weit mehr als nur ein Körperphänomen, noch um das, was oft nur noch zur Sprache bereinigt als Grundlage begrifflicher Erkenntnis verstanden wird. An der Schwelle zwischen Sinnen und Sinn ist die Rauheit der Stimme epistemologisch konstitutiv; das Denken wird als widerständige Bewegung in der Bewegung und Reibung der Stimme selbst wirklich. Es wird körperlich, sinnlich und sinnhaft zum Ausdruck gebracht. Die Potenzialität muss mithin als eben jene der Unterscheidung von aktualisiert und appräsentiert, von aktuellem und möglichen Sinn von Sinnformen selbst verstanden werden. ${ }^{13}$ Und das in jeder denkbar möglichen Konkretion.

Die Körperlichkeit des Sprechens affiziert die Worte selbst. Die Zusammenhänge der Worte zu Sätzen hängen ganz konkret an der Beweglichkeit, körper-

12 Dass es sich immer um ein Zusammenspiel der Sinne handelt, diskutiert für das Zusammenspiel von Sehen und Hören besonders spannend Doris Kolesch (Kolesch, 2006). Vergleiche aber dazu auch Chion (Chion, 1994).

13 Ich schließe hier an den phänomenologischen Sinnbegriff Edmund Husserls an [vgl. Husserl (2009), 91ff; 1991: 138ff.], wenngleich in einer notwendigen dialektischen Erweiterung, die Husserl fehlt. Zu arbeiten wäre hier mit Hegels ,Sprachphilosophie' (vgl. Hegel, 2014, S. 240-288), die die Frage nach der Entstehung der Sprache im System der Hegelschen Enzyklopädie präzise zwischen dem Bewusstsein und dem „objektiven Geist", d.h. der Gesellschaft, positioniert, aber auch Adornos „Studien zu Husserl" (Adorno, 1990), vgl. auch Eley (1984)). In beiden Fällen aber fällt das dröhnende Schweigen zur Körperlichkeit von Stimme, Sprache und Denken auf. 
lichen Gewöhnung des Mundes, der Stimmbänder, und wiederum dem eigenen Hören der Stimme im Raum und eigenen Körper ab. In seiner körperlichen Materialität ist das Sprechen situativ, konkret, empirisch. Ich erinnere meine schon gesprochenen Sätze, weil mein Mund, meine Zähne und Zunge, meine Kehle und Stimmbänder, mein Bauch, meine Lunge und meine Ohren in ihrem komplexen Zusammenspiel sich/mich an sie erinnern. Im Sprechen muss ich mich, mehr als in anderen Mitteln (auch) sprachlicher Darstellung und Ausdrucks, meinem Körper überantworten, zumindest, wenn ich nicht gegen ihn sprechen, sondern mit ihm wie mit einem Instrument die Sprache finden will. Was von Kleist unterschlägt und zu schnell auf die soziale Interaktion im Gegenüber einer Gesprächspartner:in zurückführt, ist schon im Körper des Sprechens angelegt. Zugleich ist gerade auch umgekehrt die soziale Situation der Interaktion von Bedeutung für die körperliche Wahrnehmung. Mein Körper reagiert auf die Herausforderung zu einer großen Zahl von Menschen unter Bedingungen der Anwesenheit zu sprechen. Die Körper selbst reagieren aufeinander und gestalten mein Sprechen und Hören. Auch das gilt es selbstverständlich zu bedenken und als Bedingung im Sprechen zu entwickeln. Geübte Sprecher:innen wissen nicht nur einen Umgang damit zu finden, sondern können mit dem Raum und den Körpern spielen, wissen sich die Energien zunutze zu machen, wählen Impulse aus der konkreten Situation.

Auch wenn diese Beschreibungen über die konkrete technische Vermittlung und üblichen Settings des Podcastings hinausweisen, so sind doch alle diese Fragen hier von Bedeutung. Auch technische Settings beeinflussen die körperliche Wahrnehmung des Sprechens, sei es über die Vermittlung der Stimme über Mikrofon und Kopfhörer, sei es durch Sitzposition und Körperhaltung, mein Gegenüber, den Raumklang und die Orchestrierung der vielen Handgriffe und Tätigkeiten beim Sprechen und Hören.

Über die Körperlichkeit hinausgehend und doch mit ihr in unauflösbarem $\mathrm{Zu}$ sammenhang steht aber noch ein anderer Aspekt. Für Roland Barthes ist im Hinblick auf das Sprechen der Sprache besonders ihre Unumkehrbarkeit von Bedeutung:
„Das Sprechen ist unumkehrbar, dies ist sein Verhängnis. Das Gesag- te lässt sich nicht zurücknehmen, außer durch Vermehrung: korrigie- ren heißt hier bizarrerweise hinzufügen. Beim Sprechen kann ich nie löschen, wegstreichen, annullieren; ich kann nichts anderes tun als sagen ,ich annulliere, ich lösche, ich berichtige, kurz: wieder spre- chen'." (Barthes, 2012, Herv.i.O.)

Dieses Wieder-Sprechen nennt Barthes „Gestammel“. Der skeptischen, eindeutig aus der Perspektive eines Schriftstellers verfassten Beschreibung kann ich mich hier nicht anschließen, wenngleich der beobachtete Sachverhalt bedeutsam ist. Im Unterschied zur Schrift, die zumindest seit dem Löschband der Schreibmaschine einfache technische Löschmöglichkeiten kennt, vermag das einmal Erklungene im Sprechen nicht wieder zurückgenommen zu werden. Man gerät ins Stottern, Zögern, macht Pause, ringt nach Worten, da das Sprechen nach dem Fluss und Anschluss der Kommunikation sucht. Das Rin- 
gen um Worte in der gesprochenen Sprache ist immer Teil des Geschehens selbst, immer Teil der Darstellung und Durchführung. In dieser Hinsicht liegt im Sprechen eine Notwendigkeit der Transparenz und Verpflichtung gegenüber der Entwicklung des Denkens, wo die Schrift immer wieder zur retrospektiven Abkürzung auf das vermeintlich ,Wesentliche' einlädt. Man kann dies nun Gestammel nennen und mit dem Maß der Schriftlichkeit messen, doch verkennt eine solche Darstellung die einzigartige Bedeutung des Sprechens als Mittel der Erkenntnis: Die Transparenz muss nicht verborgen, keine nachträglichen Beschönigungen vorgenommen werden, weil dies schlicht unmöglich ist. ${ }^{14}$ Wenn es aber nicht verborgen werden kann, so muss es explizit entfaltet werden; der vermeintliche Nachteil muss als Herausforderung der Entwicklung transparenter Reflexion des Denkens auch in schriftlichen Texten gedacht werden.

An dieser Stelle könnten sich nun Überlegungen zum Sprechen als Gespräch anschließen, die für die Frage nach der Bedeutung des Sprechens und Hörens für die Verfertigung wissenschaftlicher Erkenntnis von entscheidender Bedeutung sind. Dazu aber später mehr. Die hier vorgestellten Überlegungen sollen sich auf das Sprechen im Zusammenspiel von Stimme und Hören konzentrieren. Ich belasse es daher bei diesen kurzen Skizzen.

Bevor ich im letzten Teil in kurzen Zügen einige konkrete Ansätze zu wissenschaftlichen Arbeiten mit Podcasts vorstelle, möchte ich noch einmal auf die zentrale Frage zurückkommen: Welche Bedeutung haben Stimme, Hören und Sprechen für die Verfertigung der Erkenntnis? In diesem Zuschnitt auf das Sprechen unter besonderer Berücksichtigung des Mediums Podcast stellt sich also mit Holger Schulze die Frage: „How to think sonically?" (Schulze, 2017)

Die wissenschaftliche Reflexion der Erkenntnis geht üblicherweise von argumentativen, sprachlich explizierten und begrifflich gedachten Sätzen als Ziel und Leistung wissenschaftlicher Arbeit aus. Das Unverständliche verständlich zu machen, das noch nicht sprachlich Fassbare in Sprache und Worte zu begreifen, intersubjektiv zu beschreiben, verlässliches Wissens zu erzeugen und über den Zustand des bloßen Meinens und Glaubens hinauszugehen. Dabei stößt man jedoch schnell an die Grenzen des Diskurses, innerhalb wie außerhalb.

Die Grenzen des Wissbaren, die Grenzen des sprachlich-begrifflich Erfassbaren stellen auf der einen Seite die Frage: kann wirklich nur wissenschaftlich erforscht werden, was sich sprachlich-begrifflich erfassen lässt? Welche Bedeutung kommt hier etwa der sinnlich-sinnhaften ästhetischen Wahrnehmung der Körper zu, die zwar auch, aber bestenfalls nur unzureichend in Sprache gefasst werden kann. Die Bedeutung, in einem starken Sinn der Begriff der Sache, ist in jener mangelhaften Übersetzung keinesfalls gegeben. In vielen Fällen ist die sinnlich-ästhetische Wahrnehmung konstitutiv für die Erkenntnis

\footnotetext{
14 Zumindest, wenn man das Sprechen selbst ernst nimmt. Selbstverständlich ist es denkbar und gerade aufgrund dieser hochriskanten Anlage des Sprechens, der Unwägbarkeit der Wortlosigkeit oder des Gestammels mittels vorab aufgeschriebener Texte zu begegnen. Dass es sich dabei nur insofern um konzeptionell mündliches Sprechen handelt, wenn von diesem Geschriebenen abgewichen wird, wird eindrücklich in jedem ermüdenden, bloß vorgelesenen wissenschaftlichen Konferenzbeitrag erkennbar.
} 
selbst. Sprachliche Bestimmungen könnten hier nur nennen, was damit nicht reflektiert, nicht ,erfasst' wird.

Zum anderen stößt das Modell der Wissenschaftlichkeit aber auch an die Grenzen der eigenen Praxis, die, das haben etwa die ,Laborstudien', Wissenschaftsgeschichte und -soziologie in umfangreichen empirischen Studien gezeigt, keinesfalls so abläuft, wie es Forschungsberichte, Paper oder Ergebnisdiskussionen auf Konferenzen präsentieren. Die Behauptung, dass retrospektiv doch nur der kürzeste Weg zählt, der ans Ziel des Ergebnisses führt, und dieses Ziel mit der Wahrheit wissenschaftlicher Erkenntnis verwechselt, bleibt ohne jeden Nachweis ihrer Zulässigkeit: unter Auslassung des Weges bleibt die Ergebnispräsentation oft nur argumentativ mehr oder weniger geschickte Annahme.

Der Wissenschaftshistoriker und Genforscher François Jacob unterscheidet in diesem Zusammenhang Tagwissenschaft und Nachtwissenschaft:

„Wie ein Räderwerk greifen die Beweisführungen der Tagwissenschaft ineinander, und ihre Resultate haben die Kraft der Gewissheit. Ihre majestätische Ordnung lässt sich bewundern wie ein Gemälde von Leonardo da Vinci oder eine Fuge von Bach. Man kann sich darin ergehen wie in einem französischen Garten. Ihrer Vorgehensweise bewußt, stolz auf ihre Vergangenheit und ihrer Zukunft sicher schreitet die Tagwissenschaft im Licht und im Ruhm voran.

Die Nachtwissenschaft dagegen ist blindes Irren, Sie zögert, stolpert, weicht zurück, gerät ins Schwitzen, schreckt auf. An allem zweifelnd, sucht sie sich, hinterfragt sich, setzt immer wieder neu an. Sie ist eine Art Werkstatt des Möglichen, in der das künftige Material der Wissenschaft ausgearbeitet wird." (Jacob, 2000, S. 164)

Die Unterscheidung von Tag- und Nachtwissenschaft ist für die Fragen nach der Materialität der Darstellung und Durchführung des Denkens und seiner Bedeutung für die Verfertigung wissenschaftlicher Erkenntnis entscheidend. Wissenschaftstheoretisch lassen sich zwar die Probleme teilweise relativieren; die Forschung ist in Methoden und Theorie an strengen Maßstäben ausgerichtet, deren Darstellung meist unerlässlich ist und im Diskurs der Disziplinen sich der Kritik stellt, ja diese explizit sucht. Und doch ist jener krasse Unterschied zwischen Selbstverständnis, Darstellung und tatsächlicher Praxis ein Problem für das Transparenzgebot wissenschaftlicher Forschung: Um sich überhaupt der Kritik stellen zu können, muss der konkrete Prozess der Forschung selbst mit zur Darstellung gebracht werden. Wie sehr diese vermeintlich bloß wissenschaftsintern relevanten, fachlich hochspezifischen Verfahrensfragen auch für die Leistung der Wissenschaft für eine breite Öffentlichkeit von Bedeutung sind, ließ sich jüngst in der ungewöhnlich detaillierten Diskussion wissenschaftlicher Arbeit im NDR-Podcast Coronavirus-Update mit dem Virologen Christian Drosten beobachten. ${ }^{15}$ Und noch die folgenden Fragen fanden dort Erwähnung:

15 https://www.ndr.de/nachrichten/info/podcast4684.html (Zugriff am 30.07.2020). 
„Sobald ein Wissenschaftler dann einen Artikel schreibt, um das Resultat seiner Arbeit zu veröffentlichen, vergisst er, bewusst oder unbewusst, die Nachtwissenschaft, um nur noch von der Tagwissenschaft zu sprechen. Nun geht es darum, Ordnung in eine Masse von Daten zu bringen, die im Laufe der Monate und Jahre gesammelt worden sind. Ihnen eine Form zu geben, eine vernünftige Geschichte aus ihnen herauszuholen, die dann den offiziellen Forschungsbericht darstellen wird."(Jacob, 2000, S. 165)

Man muss hier nicht einmal Täuschungsabsicht unterstellen. Das Problem liegt vielmehr schon im Medium der Schrift selbst - zumindest mitbegründet:

„Wahrhaftig eine seltsame Übung. Wissenschaft ist vor allem eine Welt von Gedanken in Bewegung. Die Niederschrift bringt diese Gedanken zum Stillstand; lässt sie erstarren; als wollte man ein Pferderennen mit einer Momentaufnahme wiedergeben." (Jacob, 2000, S. 166)

Gerade hier bieten Podcasts und auditive Medien, die Durchführung als Darstellung der Verfertigung wissenschaftlicher Erkenntnis in Stimme, Sprechen und Hören noch weitgehend ungenutzte, praktisch kaum entwickelte Möglichkeiten. Nicht nur lässt sich das Ringen um die Erkenntnis als Stammeln, das Zögern und Stolpern der Nachtwissenschaft selbst zur Sprache, zur Stimme verhelfen; entscheidend ist hier, wie oben diskutiert vor allem die körperliche Dimension wissenschaftlicher Erkenntnis. Damit ist natürlich nicht gemeint, dass alle wissenschaftliche Erkenntnis notwendig körperliche Qualität hat oder auch nur auf jene Darstellungsformen angewiesen ist. Zugleich aber sind in fast jeder wissenschaftlichen Arbeit Körper und sinnlich-ästhetische Wahrnehmungsprozesse so eng eingeflochten, dass sie nur unter Verlust der Transparenz und Nachprüfbarkeit und gar der sprachlich-begrifflichen Leistungen der Arbeit selbst außer Acht gelassen werden können.

How to think sonically? (Schulze, 2017) Genauer noch in dem von mir diskutierten Fall: wie mit der Stimme, dem Sprechen und Hören denken? Wenn ich etwas wie eine Zusammenfassung der oben genannten Aspekte versuchen kann, dann schlage ich vor: a) die Bedeutung der Stimme, des Sprechens und Hörens in den heute üblichen Verfahren der Verfertigung wissenschaftlicher Erkenntnis ernst zu nehmen und zu reflektieren. b) Der Stimme als Medium der Erkenntnis im Sprechen und Hören kommt hier eine besondere Bedeutung für eben jene notwendige Reflexion der Materialität der Erkenntnis zu. c) Der Überschuss, das Mehr der Stimme im Sprechen und Hören, die Unbegrifflichkeit stimmlicher Erkenntnis weist über Diskurse, Argumente, etablierte Verfahren hinaus und konfrontiert mit der Nichtidentität der Begriffe und ihrer Gegenstände. d) Das Medium der Stimme weist auf die Medialität wissenschaftlicher Erkenntnis zurück und zugleich darüber hinaus. Eine Entwicklung der Stimme als etabliertes Mittel der Erkenntnisproduktion statt nur ihrer Vermarktung ist damit schon von entscheidender medientheoretischer wie wissenschaftstheoretischer und -praktischer Relevanz. e) Die Körperlichkeit der Erkenntnis findet in der Stimme ein Mittel der Darstellung 
und Durchführung, die als sinnlich-ästhetische, körperliche Weise neue Arten des Wissens hervorbringen kann.

\section{Podcasting als Medium der Wissenschaft}

Im letzten Teil meines Beitrags möchte ich lediglich drei Bereiche nennen, als dass ich sie eingehend diskutieren könnte. In diesen soll die Bedeutung des Podcastings im Kontext von Stimme, Sprechen und Hören für wissenschaftliche Erkenntnisproduktion erkennbar werden. Wie können Stimme, Hören und Sprechen als Medien der Durchführung und Darstellung wissenschaftlicher Erkenntnis selbst verstanden werden? Wie lässt sich das konkret gestalten? Inwiefern können und müssen Podcasts als Form der Vermittlung auditiver Beiträge nicht nur als Medium der public science, sondern als genuines Medium wissenschaftlicher Erkenntnisproduktion und des Diskurses selbst verstanden und entwickelt werden?

Es mag der Eindruck entstehen, dass die bisherigen Überlegungen meines Beitrags unverbunden zu den folgenden Umsetzungen stehen, doch sollen erste eher als Schlüssel zum nun Folgenden verstanden werden. Ich hoffe zumindest einige der Bezüge deutlich machen zu können.

\subsection{Recording Culture}

Die erste hier vorzustellende Arbeit ist „Recording Culture“ von Daniel Makagon und Marc Neumann (2009). Die Sammlungen und Studien widmen sich alle in der ein oder anderen Form der Arbeit mit Sound, Musik, Stimmen oder anderen auditiven Phänomenen. Disziplinär der Kultur- und Sozialanthropologie zuzuordnen, entwickeln die beiden Autoren hier die Methodenkritik der Writing Culture Debatte (vgl. Clifford, 1986a) für die Arbeit mit Audioaufnahmeverfahren weiter. Diese so wesentliche Debatte für die Ethnografie und Mitauslöser der cultural turns der Geisteswissenschaften stellte die kritische Frage nach der Verfertigung der Kultur im Schreiben wissenschaftlicher Texte. Daran anschließend müssen heute die falschen Dualismen von Forschung und Produktion und Prozess und Produkt neu kritisiert werden (Makagon \& Neumann, 2009, S. 6).

Anhand früher und aktueller experimenteller Audio Recording-Produktionen diskutieren die Autoren beeindruckende Beispiele aus so diversen Bereichen wie der frühen ,Musikethnographie' von Vater John und Sohn Alan Lomax in der ersten Hälfte des 20. Jahrhunderts (vgl. Makagon \& Neumann, 2009, S. 5ff) oder den Interviews und Gesprächen von Tony Schwartz zu Urbanismus, Migration und Milieuforschung (ebd., S. 10-16) bis hin zu ,Soundscapes' Forschung (ebd., S. 27ff.), ,Sound walks' (ebd., S. 32-39), ,Radio Diaries' und ,Audio Essays' (ebd., S. 39ff.) oder ,Audio Documentaries' (ebd., S. 42ff.) als Formen der, Sonic compositions'. Auch wenn ich hier die Studien nicht detailliert diskutieren kann, so muss doch deutlich auf die Zusammenhänge zu den Fragen dieses Beitrags verwiesen werden: Bis heute nur rudimentär diskutierte 
Möglichkeiten der Audio-Recordings und auditiven Darstellungen für die Untersuchung von Milieus auch als Sprachgemeinschaften, den Klang der Stimme als kritisches Medium gerade jener kulturwissenschaftlichen Disziplinen, die sich der Aufgabe stellen, marginalen Milieus, Gruppen, Ethnien, Berufsgruppen, etc. eine Stimme zu geben, ja ihre Stimme ernst nehmen zu müssen, werden in ihrer Dringlichkeit dort - wenngleich in Form eines geschrieben Textes - verhandelt. Bei der Diskussion der Beiträge fällt dann auch auf, dass einer erkenntnistheoretischen Reflexion auditiver Phänomene in der Wissenschaft auf grundlegender Ebene auch hier noch immer die Aufmerksamkeit fehlt, die sie verdient.

Ebenfalls muss an die Unterscheidung von Tag- und Nachtwissenschaft erinnert werden. Im Anschluss an die Writing Culture Debatte gelingt es besonders der Ethnographie und methodisch verwandten kulturwissenschaftlichen Disziplinen, die gängige strikte Unterscheidung von Forschung und Ergebnis als wissenschaftlich problematisch zu reflektieren. Die hörbaren Phänomene selbst drängen über das bloß Sprachlich-Begriffliche hinaus, verweisen auf die in auf den geschriebenen Text zielenden Wissenschaftskulturen tauben Ohren (statt blinder Flecken). Die Widersprüche zwischen Material und Reflexion, vermittelt durch die sinnliche Wahrnehmung der Forscherinnen selbst und aufbereitet als eine zu erzählende Geschichte, müssen in ihrer Bewegung, im Prozess als ihre Entfaltung dargestellt werden.

Stephan Porombka und Holger Schulze haben über diese Fragen in einem Gespräch zum Verhältnis von Text und Audio nachgedacht (vgl. Porombka \& Schulze, 2018a, S. 2018b). Wenn man die vermeintliche, bisweilen ideologisch verfestigte Alternativlosigkeit schriftlicher Ergebnisdokumentation in der Wissenschaft bedenkt, bieten Podcasts und auditive Formate im Allgemeinen eine einzigartige Möglichkeit, diese Verwachsungen aufzubrechen. „Der Effekt wäre, dass der gedruckte Text auditiv verflüssigt wird" (Porombka \& Schulze, 2018b, S. 273); gerade das Vorläufige, Brüchige, Unsichere und Riskante der Forschung, das sich oft genug in hörbaren statt in lesbaren Notizen und Gedanken zeigt, könnte ,freigelegt' werden: „Die auditive Schicht des Forschens [...] als auditive zugänglich gemacht werden" (ebd., S. 272).

Im Zusammenhang mit den oben skizzierten epistemologischen, theoretischen und methodologischen Fragen muss jedoch mit Porombka und Schulze darauf hingewiesen werden, dass es dafür praktisch noch keine entwickelte Form gibt:

„Im Grunde muss die Präsentation dieses Aggregatzustandes überhaupt erst entwickelt werden. Und zwar notwendigerweise über ein Ausprobieren und Erweitern von Möglichkeiten. Das Freilegen der auditiven Schicht könnte man ziemlich weit treiben. Hineinnehmen würde man Gespräche, Abstimmungsfragen, vielleicht auch kleine Audionotizen, Gedankenfetzen, Monologe, sprechendes Nachdenken, das Einschalten des Aufnahmegeräts und das freie Assoziieren während des Spazierengehens vor dem Einschlafen, wenn man nachts aufwacht, also Audionotizen". (Porombka \& Schulze, 2018b, S. 274) 
Sowohl im Bereich der Wissenschaft zu ohnehin auditiven Phänomenen als auch im Kontext geistes- und kulturwissenschaftlicher Arbeit darüber hinaus, können solche Formate, sofern sie einmal entwickelt werden, einen eigenen Beitrag zum wissenschaftlichen Diskurs bieten. Nicht zuletzt einen kritischreflexiven. Denn: die Entwicklung auditiver Formen wissenschaftlicher Reflexion im Sinne der Recording Culture oder der Textur des „Textgeredes“ im Anschluss an Porombka und Schulze ermöglicht auch, den schriftlichen Text in seiner Funktion und Bedeutung neu zu denken (siehe dazu auch den letzten Abschnitt weiter unten zum „Sprechenden Denken“).

\subsection{Das Audio Paper-Manifest}

Ein weiterer und meines Erachtens entscheidender Bereich der notwendigen Entwicklung von Podcasts als Medium wissenschaftlicher Erkenntnis statt nur ihrer Vermarktung und Verbreitung ist der Bereich der sogenannten künstlerischen Forschung. In den letzten Jahrzehnten sind in diesem Bereich eine ganze Reihe an Arbeiten entstanden, zahlreiche Konferenzen durchgeführt und Arbeiten veröffentlicht worden. ${ }^{16}$ Er verbindet im strengen Sinn ein kritisches Bestreben nach einer Form ästhetischer Erkenntnis, mit einem Ringen um neue Formen des Wissens, Fragen der Praxis, Performanz und Darstellung sinnlich-ästhetischer Erkenntnis, einer neuen Reflexion der Bedeutung des Körpers und der Materialität der (ästhetischen) Erfahrung der Forscher:innen, und „Geltungsfragen der ästhetischen Erkenntnis selbst, [...] ihre[r] Begründung , als Wissen'. " (Mersch, 2015, S. 7)

Die Ansätze künstlerischer Forschung führen eine explizite Kritik positivistisch, mechanistischer oder anders dominant naturwissenschaftlich verpflichteter Leitmodelle wissenschaftlicher Erkenntnis, welche in ihrer großen gesellschaftlichen Relevanz und wirtschaftlichen Nutzbarkeit auch innerhalb wissenschaftlicher Diskurse kaum in der nötigen Strenge kritisiert werden können. Ganz im Sinne der „Kraft der Kunst“ (vgl. besonders Menke, 2014) muss dabei die ästhetische Kraft als kritisches Potenzial eines ,vernünftig rationalen' Diskurses verstanden werden. Sinnlich-ästhetische Kräfte vermögen diskursive Strukturen infrage zu stellen. Gemeint ist hier ganz wie oben eben jene Kraft des über das sprachlich-begriffliche Hinausgehenden, welches ich mit Betonung auf die Stimme besprochen habe.

Ein neuer Beitrag zu Fragen, Möglichkeiten und Herausforderungen künstlerischer Forschung im Bereich auditiver Phänomene und Formen wissenschaftlicher Reflexion im Medium von Audio ist hier „Audio Papers - a manifesto" der beiden Sound Studies und Musikwissenschaftlerinnen Sanne Krogh Groth und Kristin Samson (Groth \& Samson, 2016, vgl. auch 2019). In ihrem Manifest fordern und erklären sie, dass es Zeit sei, der dominanten schriftlichen Form der Verfertigung von wissenschaftlicher Erkenntnis eine andere, hörbare Form wissenschaftlicher Veröffentlichungen entge-

16 Vgl. hierzu zahlreiche Handbücher, Monografien, Manifeste oder Paper, von denen ich hier nur die folgenden nennen möchte: Badura et al. (2015); Bippus (2019), 2015; Haarmann (2019); Henke, Mersch, Van der Meulen, Strässle \& Wiesel (2020); Mersch (2015); Schenker (2012). 
genzustellen, gerade für hörbare Phänomene und Formen der Erkenntnis, die das Hören betreffen ${ }^{17}$. Audio Papers sollen verstanden und entwickelt werden als idiosynkratische (weil an die jeweilige Forscherin notwendig gebundene), performative, sinnlich-ästhetische, (räumlich, sozial, kulturell, historisch) situierte, partielle ${ }^{18}$ Darstellungen und Werke mit dem Anspruch wissenschaftlicher, um ,Wahrheit' streitender Erkenntnis. Sie kritisieren die Vorstellung, dass nur als Erkenntnis und Wissen zählen kann, was notwendig sprachlich, am besten schriftlich und in einem solchen Sinne begrifflich zur Darstellung gebracht werden kann. Audio Papers können hier als ein adäquates Mittel verstanden werden, um die widerständige Materialität (hörbarer) Phänomene ernst zu nehmen, ihre Bedeutung für die Erkenntnis als sinnlich wahrnehmbare zu belassen, ohne diese in sprachliche Formen übersetzen zu müssen. Oft genug scheitern solche Übersetzungen nämlich gerade in der darin behaupteten Allgemeingültigkeit oder der Rückübersetzbarkeit, oder fast immer: der Anschaulichkeit.

So erschreckend ${ }^{19}$ neu das Manifest zum Format des Audio Paper auch sein mag und so wenig es bislang konkrete Entwicklung erfahren hat, ${ }^{20}$ stehen die schon verfassten, oft im wissenschaftlichen Diskurs marginalisierten Audio Paper oder Vorformen dennoch in einer Ökologie anderer Formen und Ansätze. Zu nennen wären hier etwa künstlerische Avantgarde-Bewegungen des 20. Jahrhunderts, Radiohörspiele, Sound Poetry, Murray Schafers bekannter Text zu Soundscapes (Schafer, 1993), Sound Walks und zahlreiche andere Formate (vgl. Groth \& Samson, 2019, S. 190f).

Auf die Frage Holger Schulzes zurückkommend, How to think sonically? (Schulze, 2017), muss das Audio Paper als erster ernsthafter, den Gattungen wissenschaftlicher Diskurse auch formal gerecht werdender Vorstoß verstanden werden. Wie streng und genau und doch an der Grenze der als wissenschaftlich akzeptierten Formen der Reflexion eine solche Arbeit durchzuführen ist, wird vielleicht erst in aktuellen Skizzen und Manifesten erkennbar. Die Entwicklung von Formen des Wissens, des Ringens um wissenschaftliche Wahrheit und Erkenntnis jenseits der sprachlich- begrifflichen Maßstäbe, lässt sich dann auch nicht wirklich in einem solchen Beitrag wie meinem darstellen. Dass gerade Podcasts in diesem Bereich eine besondere Bedeutung erfahren, ist jedoch evident. Die intime Hörsituation über Kopfhörer ermöglicht ein intensives und sehr genaues Hören. Die Länge ist nicht durch Sendezeiten oder (nur in Extremen) durch Speicher oder Bandbreite begrenzt und die Vielfalt der (möglichen) Formate ist groß. Experimentelle, auf sinnlich-ästhetische Wahrnehmung rekurrierende Darstellungen der Audio Papers sowie Ansätze künstlerischer Forschung lassen sich gerade im Medium der Podcasts umsetzen. Die Frage bleibt nur, wie lange es dauert, bis

17 Etwa alle Bereiche der Musik, der Klangforschung, Sound Studies, des Sound Design oder der Klangund Musikanthropologie.

18 Ganz im Sinne der "Partial Truths“ (Clifford, 1986b) der Writing Culture Debatte.

19 Erschreckend, weil die medientechnologischen Bedingungen für solche Formate schon längst, wenngleich nicht in der Einfachheit und Ubiquität gegeben waren, aber diese Bedingungen halten andere Disziplinen auch nicht von der Nutzung aufwändiger neuer Technologien ab.

20 Bis auf wenige Seminare, Konferenzen, Online-Zeitschriften, und Forschungsschwerpunkte einzelner steht die Entwicklung erst ganz am Anfang. Der mehrfach beobachtete Podcast-Boom hat diese Fragen noch kaum erfasst. 
jene Formen etabliert sind und die Verbreitung finden, die sie verdienen.

Audio Papers als wissenschaftliche Podcasts müssen, so mein Vorschlag, als ein wesentlicher Beitrag und eine Antwort auf genau die Frage in aller notwendigen wissenschaftlichen Strenge verstanden werden: How to think sonically?

\subsection{Sprechendes Denken}

Der letzte Gegenstand, an dem ich die Bedeutung von Stimme, Sprechen und Hören als Mittel der Verfertigung, Darstellung und Durchführung wissenschaftlicher Erkenntnis zu zeigen versuche, ist nun das, was ich versuchsweise „Sprechendes Denken*“* genannt habe (vgl. Klenk, 2020). Es geht zurück auf ein „Experimentalsystem” (Rheinberger, 1992), welches ich im Jahr 2017 entwickelt habe, und das in seiner Erweiterung schließlich ein Dissertationsprojekt wurde. Zugrunde lag der Entschluss, einen täglichen Podcast als eine Art gesprochenes Denk- und Arbeitstagebuch zu führen. In 365 Folgen von unterschiedlicher Länge entwickelte ich dann eine Arbeitsform im Medium der Selbstgespräche, technisch vermittelt durch Mikrofon, Kopfhörer und Computer, hochgeladen und laufend veröffentlicht unter der URL https://podlog.noradio.eu.

In dieser Arbeit entwickelte sich für mich überhaupt erst ein Bewusstsein für eben jene Zusammenhänge von Stimme, Sprechen und Denken, wie ich sie in diesem Beitrag zu skizzieren versuche. Eine solche Arbeitsweise erzwingt die Einsicht, dass wissenschaftliches Denken nicht als etwas von seiner Darstellungsform Getrenntes betrachtet und nicht von den Fragen der Durchführung und Praxis jenes wissenschaftlichen Arbeitens unterschieden werden kann. So ist dann auch die Arbeit selbst konsequent eine Art selbstreflexive Arbeit zum Sprechenden Denken und damit zur Form der Arbeit, statt nur zu ihrem Inhalt, zur Arbeit in Experimentalsystemen an experimentellen Fragen zu Durchführung und Darstellung der Verfertigung wissenschaftlicher Erkenntnis geworden.

Was in dem bisher Geschriebenen zu Stimme, Sprechen und Hören noch keine Erwähnung fand, ist hier zentral, nämlich das Denken im Gespräch (und Selbstgespräch). In der Philosophie auf eine lange Tradition zurückblickend, ist zumindest der Begriff des Gesprächs noch heute von entscheidender Bedeutung in der epistemologischen Reflexion von Erkenntnis. Das Gespräch als Metapher der Dialektik der Erkenntnis, als Dialogik, als Modell des Ringens um Erkenntnis, als Abwehr eristisch-sophistischer Rechthaberei und Rhetorik zum Selbstzweck, ist der Dominanz der Schrift zum Trotz ein wirkmächtiges Bild. Aber ist dies alles, was möglich ist?

Als konkretes Philosophisches oder Wissenschaftliches, d.h. im allgemeinen als eines auf Erkenntnis Zielendes, ist das Gespräch ein gemeinsames Ringen um die Wahrheit im Medium von Rede und Gegenrede, Frage und Antwort, Satz und Gegensatz, Urteil und Anschluss, Vorschlag und Weiterentwicklung, Assoziation und Rezeption, Einwurf, Abschweifung, Exkurs, voller Brüche 
und Pause, Schlaufen der Rekursion, und vielen Formen mehr. ${ }^{21}$ Es darf dabei keinesfalls mit dem Modus der Argumentation verwechselt werden. Die Argumentation ist im besten Fall Mittel zur hinreichend schlüssigen Darstellung eines Urteilszusammenhangs unter Ausschluss anderer Möglichkeiten, im schlimmsten Fall bloßes Mittel sich (oder die eigene Position) zu behaupten. Die tatsächliche Arbeit, gar das gemeinsame Ringen um Erkenntnis und Wissen ist hierfür nicht notwendig. Das Gespräch als Mittel der Erkenntnis dagegen ist notwendig freier, sowohl im Hinblick auf seine Zwecke als auch auf seine je konkreten Entwicklungen. Ich komme darauf zurück.

Selbstverständlich sind Gespräche auch außerhalb ihrer Bedeutung als Metapher für die Aushandlung (wissenschaftlicher) Erkenntnis in Lehre und Forschung allgegenwärtig, doch spielt sie für die schriftlichen Texte dann kaum mehr eine Rolle. Die Praxis verschwindet hinter dem Ergebnis; über die Nachtwissenschaft wird geschwiegen, wenn das strahlende Licht der Texte den kürzest möglichen Weg als den der Wahrheit vorgibt. Die hierbei wie oben bereits diskutierten Probleme mangelnder Transparenz und Nachvollziehbarkeit verschwinden im Wechsel des Mediums der Darstellung und Durchführung: vom Gespräch zum schriftlichen Text verliert sich der Weg. Doch gilt für Wissenschaft gerade in dieser Hinsicht, was Jürgen Mittelstraß im Zusammenhang mit dem philosophischen Gespräch für die Philosophie sagte: „Philosophie lässt sich nicht sagen (in Form von Lehrbuchwissen), sondern nur tun (in Form von philosophischen Dialogen bzw. einer verwirklichten Orientierung)." (Mittelstraß, 1982, S. 151)

Gespräche können jedoch nicht nur als Praxis, sondern zugleich als Gattung und Form betrachtet werden. Als Medium der Erkenntnis haben sie eine eigene Struktur, eigene Bedingungen des Denkens, stellen einen eigenen Raum an Möglichkeiten. Im Hinblick auf die hier besprochenen Fragen von Stimme, Sprechen und Hören sind dabei noch einige Aspekte von besonderer Bedeutung. Insofern Gespräche sich im Medium des Sprechens und Hörens der Stimmen als körperliche Praxis, sinnlich-sinnhaft vermittelt auf die jeweiligen Gesprächspartner:innen beziehen, verweisen sie darin über die diskursiv bestimmten, disziplinär limitierten oder theoretisch und methodisch gefassten ,Inhalte' des Gesprächs hinaus. Gespräche schaffen im Zusammenspiel der Stimmen als gesprochene und gehörte, d.h. jeweils körperliche, ästhetisch-sinnlich vermittelte einen eigenen Raum. ${ }^{22}$ Dieser Raum steht sozusagen orthogonal zur Struktur wissenschaftlicher Diskurse (vgl. Klenk, 2020, S. 77f), oder - noch schärfer - Gespräche können gerade dadurch, dass sie einen eigenen Raum, eine eigene Situation herstellen, die ,Ordnung der Diskurse' suspendieren. Mit Karlheinz Stierle kann man

21 Wenn ich hier und im Weiteren von Gespräch schreibe, dann ist damit das Selbstgespräch, sofern es kein Monologisieren ist, immer mit gemeint. Gerade in der technischen Vermittlung beim Podcasting d.h. im Normalfall dem Sprechen in Mikrophone und dem gleichzeitigen Abhören über Kopfhörer, ist die Gesprächssituation auch mit sich selbst gegeben. Oben ausgeführte Zusammenhänge zwischen Stimme, Sprechen und Hören bestimmen auch die Dynamik des Selbstgesprächs. Für genauere Untersuchungen zu den philosophischen Bedingungen des Selbstgesprächs als Gespräch und Mittel der Erkenntnis siehe auch Klenk (Klenk, 2020, S. 47ff)

22 Die Räumlichkeit ist hier mehr als nur Metapher eines Denkraumes, spielt sie doch ganz wesentlich auch auf die notwendige Räumlichkeit aller auditiver Phänomene an. Klang und Hören sind immer räumlich (vgl. Schulze, 2017). 
feststellen:

„Im Diskurs wird die ursprüngliche Weltoffenheit des Dialogs auf Formen der Vereindeutigung, Geschlossenheit hin überschritten. Gerade angesichts des absoluten, monologischen Anspruchs der Diskurse wird aber das Gespräch zu einer neuen Erfahrung der Entlastung, zugleich auch der Öffnung diskursiver Geschlossenheit und dogmatischer Enge. Die Verhexung des Verstands durch den Diskurs, die darin liegt, den Diskurs selbst nur immer weiterzutreiben, institutionell und diskursiv abgesicherte Segmentierungen absolut zu setzen, lässt dem, der sich ihr zu entwinden trachtet, die Rückkehr zur ursprünglichen Offenheit und elementaren Wahrheit der im Gespräch zu sich kommenden Einsicht als Alternative von einem fast unwiderstehlichen Reiz erscheinen." (Stierle, 1996, S. 306)

Was hier durchaus als Phantasma einer Ursprünglichkeit romantisiert erscheint, kann mit den Überlegungen zu Stimme, Sprechen und Hören ganz konkret gefasst werden. Das immer Körperliche, Ästhetisch-Sinnliche, der Überschuss der Stimme im Sprechen und Hören sprengt die „Verhexung des Verstands" durch die Ordnung des Diskurses; die Ordnung des Diskurses kann in der Situation des Gesprächs aufgehoben, suspendiert und über sich hinaus verwiesen werden. Schon das Stammeln, Abbrechen, immer wieder neu ansetzen im Sprechen und Hören, die körperlichen Erinnerungen des Mundes, der Ohren, des ganzen Körpers fügen sich nicht den wissenschaftlichen Operationalisierungen. Wenn es zudem im Gespräch selbst um Fragen erkenntnistheoretischer Bedeutung von Sinn und Sinnlichkeit geht, von Stimme, Sprechen und Hören, wird die Materialität des Gegenstands wie des Denkens selbst in die Darstellung eingeführt. Genau diese Kraft des Gesprächs muss als Antwort auf die hier selten gestellte Frage, wie neue Erkenntnis möglich wird, verstanden werden. Die Eindringlichkeit lässt einen wundern, dass dies für ein modernes dialektisches Denken noch kaum entfaltet wurde.

Unter diesen Bedingungen kann dann auch gelingen, was ebenfalls mit Stierle als ein wesentliches Merkmal von Gesprächen formuliert werden kann: die freie Fortführung. Die freie Fortführung als Wesen des Gesprächs hält und stabilisiert sich selbst in der „Kontinuität der Hinwendung“ auf die Sache. Gespräche sind in ihrer Entwicklung offen, mithin - so möchte ich es zumindest für diesen Beitrag zuspitzen - weil sie der Widerständigkeit der Körperlichkeit der Stimme nie ganz entkommen.

„Das Gespräch, die Konversation, der Dialog sind zunächst bestimmt durch die Kontinuität einer Zuwendung der durch dies symbolische Verhältnis einander zugeordneten Personen. Was das Gespräch aber in seiner Besonderheit bestimmt, und insbesondere von der Konversation abhebt, die der gesellschaftliche horror vacui erzwingt, liegt über die Kontinuität wechselseitiger Zuwendung hinaus in der gemeinsamen Hinwendung auf eine Sache, ein Verhältnis, ein Problem." (Stierle, 1996, S. 301) 
Zuletzt möchte ich die naheliegende Frage nach dem Subjekt des Gesprächs hervorheben. Mit Jürgen Mittelstraß lässt sich feststellen: „Im philosophischen Dialog stehen nicht irgendwelche Meinungen oder Probleme, sondern die Subjekte der philosophischen Wissensbildung auf dem Spiel. Darin beruht der agonale und der Sokratische Charakter dieser Dialogform." (Mittelstraß, 1982, S. 139) Was hier Subjekt heißt, darf jedoch nicht vorschnell als Kommunikationspartner:in missverstanden werden. Subjekt ist die Reflexion selbst, Denken als Praxis ist in dieser erst Subjekt.

Hier ist nun ein entscheidender Bezug zu den Fragen der Subjektivität des Hörens herzustellen. Mit Nancy, Voegelin und Barthes (s.o.) ist es genau jene Subjektivität des Gesprächs, die hier im Medium der Stimme, des Sprechens und Hörens , auf dem Spiel steht' (im besten aller Sinne). Die Unterscheidungen von Innen und Außen, von Subjekt und Objekt, von sinnlich-ästhetischer Wahrnehmung aufeinander bezogener Körper und dem materiellen Überschuss der Stimme verweisen die Unterscheidungen auf sich selbst, führen eine Widerständigkeit als Bedingung dialektischer Erkenntnis ein. So wie die Materialität und Rauheit der Stimme sowohl auf ihre Medialität als auch das immer schon Zuviel verweist, so steht die Stimme sowohl für die Subjektivität als auch die Infragestellung der Subjektivität zugleich. Nicht zuletzt darin liegt mithin ihre eigene noch weiter zu entwickelnde Bedeutung für die Möglichkeiten der Reflexion der Verfertigung wissenschaftlicher Erkenntnis im Sprechen und Hören.

\section{Fazit}

Kaum eine Form auditiver Inhalte bestimmt das Medium Podcast so wie das ,natürliche', freie Gespräch. Wie ich mit diesen kurzen Skizzen versuche zu zeigen, gewinnt dieses aber gerade im Kontext der Wissenschaft noch einmal zusätzlich an Bedeutung: Wissenschaftliche Arbeit besteht - vielleicht sogar zum größten Teil - aus und in Gesprächen, nur fehlt bislang nach wie vor eine ernsthafte erkenntnistheoretische und methodologische Reflexion über das Führen von Gesprächen als Mittel der Erkenntnis unter aktuellen technischen und medialen Bedingungen. Das Gebot der schriftlichen Darstellung bringt alle Gespräche zum Verstummen. Die Stimme für die Stimme erhebend, schreibe ich diesen Beitrag als Aufruf zur Weiterentwicklung dieser Überlegungen. Es kann dabei aber gerade nicht zu einer Rückkehr des Gesprächs, des Mündlichen vor der Erfindung der Schrift oder gar um eine neue Form der Technikkritik gehen, sondern im Gegenteil um die iterative, dialektische, kritische Weiterentwicklung von Darstellung und Durchführung wissenschaftlicher Erkenntnis. Die Abstraktionsgewinne, die Schärfe, Präzision, einfache Verbreitung und etablierte Form schriftlicher Kommunikation kann in ihrer Bedeutung für die Entwicklung wissenschaftlich komplexen Denkens kaum überschätzt werden. Wenn man heute daher eine Entwicklung des Gesprächs als Mittel der Erkenntnis fordert, dann muss damit eine Entwicklung vor diesem Hintergrund gemeint sein. 
In verwandter, ideologiekritischer Hinsicht schrieb Adorno im Aphorismus 65 der Minima Moralia:

„Wenn die Schriftsprache die Entfremdung der Klassen kodifiziert, dann lässt diese nicht durch Regression auf die gesprochene sich widerrufen, sondern nur in der Konsequenz der strengsten sprachlichen Objektivität. Erst das Sprechen, das die Schrift in sich aufhebt, befreit die menschliche Rede von der Lüge, sie sei schon menschlich." (Adorno, 2003b, S. 115)

Um diese Entwicklung eines Sprechens (und Hörens) muss es heute gehen. Wenn ich über Stimme, Sprechen und Hören im Medium des Podcasting als wissenschaftliches Mittel der Verfertigung von Erkenntnis schreibe, geht es mir genau darum. Nicht zurück zum Körper, den Sinnen, dem Sprechen und Hören, dem Gespräch, statt der Rationalität, dem Sinn, dem Schreiben und Lesen, und dem Diskurs. Vielmehr gilt es, Podcasts in der Wissenschaft als Wissenschaft zu entwickeln: als sinnlich-ästhetische Formen des Wissens und künstlerische Forschung, als Körperwissen in der Vermittlung und Darstellung von Sinn und Sinnlichkeit; als Medium der Transparenz, Methodenreflexion und konstruktive Praxistheorie von Wissenschaft, als Darstellung der Praxis der Forschung und dieser verpflichtet; und schließlich in der Entwicklung eines „,Sprechenden Denkens“ als dialektische Durchführung durch Darstellung, als Denken im Gespräch.

\section{Referenzen}

Adorno, T. W. (1990). Zur Metakritik der Erkenntnistheorie. Studien über Husserl und die pänomenologischen Antinomien (suhrkamp taschenbuch wissenschaft). In R. Tiedemann (Hrsg.), Gesammelte Schriften, Band 5: Zur Metakritik der Erkenntnistheorie Drei Studien zu Hegel (3. Aufl., S. 7-245). Frankfurt am Main: Suhrkamp.

Adorno, T. W. (2003a). Gesammelte Schriften, Band 16: Musikalische Schriften I-III: Klangfiguren; Quasi und fantasia; Musikalische Schriften III (suhrkamp taschenbuch wissenschaft) (1. Aufl.). Frankfurt am Main: Suhrkamp.

Adorno, T. W. (2003b). Gesammelte Schriften, Band 4: Minima Moralia: Reflexionen aus dem beschädigten Leben (suhrkamp taschenbuch wissenschaft) (1. Aufl.). Frankfurt am Main: Suhrkamp.

Bachmann-Medick, D. (2009). Cultural turns: Neuorientierungen in den Kulturwissenschaften (Rororo) (Band 55675 : Ro). Reinbek bei Hamburg: RowohltTaschenbuch-Verl.

Badura, J., Dubach, S., Haarmann, A., Mersch, D., Rey, A., Schenker, C. et al. (Hrsg.). (2015). Künstlerische Forschung: ein Handbuch (1. Auflage.). Zürich Berlin: Diaphanes. 
Barthes, R. (2012). Das Rauschen der Sprache (Kritische Essays). In D. Hornig (Übers.), Das Rauschen der Sprache (3. Aufl., Band 1695, S. 88-91). Frankfurt am Main: Suhrkamp.

Barthes, R. (2015a). Die Rauheit der Stimme (edition suhrkamp). In D. Hornig (Übers.), Der entgegenkommende und der stumpfe Sinn. Kritische Essays III (9. Auflage., S. 269-278). Frankfurt am Main: Suhrkamp.

Barthes, R. (2015b). Die Musik, die Stimme, die Sprache (edition suhrkamp). In D. Hornig (Übers.), Der entgegenkommende und der stumpfe Sinn. Kritische Essays III (9. Auflage., S. 279-285). Frankfurt am Main: Suhrkamp.

Barthes, R. (2015c). Zuhören (edition suhrkamp). In D. Hornig (Übers.), Der entgegenkommende und der stumpfe Sinn. Kritische Essays III (9. Auflage., S. 249-263). Frankfurt am Main: Suhrkamp.

Bippus, E. (2019, November 24). Potenziale künstlerischer Forschung*. mdwMagazin. Zugriff am 17.6.2020. Verfügbar unter: https://www.mdw.ac.at/ magazin/index.php/2019/11/24/potenziale-kuenstlerischer-forschung/

Blumenberg, H. (2007). Theorie der Unbegrifflichkeit. (A. Haverkamp, Hrsg.). Frankfurt am Main: Suhrkamp.

Butler, J. (1997). Excitable speech: The politics of the performative. New York, London: Routledge.

Chion, M. (1994). Audio-vision: Sound on screen. (C. Gorbman, Übers.). New York: Columbia University Press.

Clifford, J. (Hrsg.). (1986a). Writing culture: The poetics and politics of ethnography; a School of American Research Advanced Seminar [...] XA-GB. Berkeley, Calif. [u.a.: Univ. of California Press.

Clifford, J. (1986b). Introduction: Partial truths. In J. Clifford (Hrsg.), Writing culture: The poetics and politics of ethnography; a School of American Research Advanced Seminar [...] XA-GB (S. 1-26). Berkeley, Calif. [u.a.: Univ. of California Press.

Dolar, M. (2011). The vocal stone (Eikones). In M. Butte \& S. Brandt (Hrsg.), Bild und Stimme (S. 31-47). München: Wilhelm Fink.

Dolar, M. (2014). His Master's Voice: eine Theorie der Stimme (Suhrkamp Taschenbücher Wissenschaft). (M. Adrian \& B. Engels, Übers.) (1. Auflage.). Berlin: Suhrkamp.

Eley, L. (1984). Konstruktive Phänomenologie und kritische Theorie. Adornos Kritik der transzendentalen Phänomenologie Husserls. Eine Anmerkung zu Heideggers Seinsfrage. (Uni-Taschenbücher). In J. Naeher (Hrsg.), Die Negative Dialektik Adornos. Einführung - Dialog (S. 59-89). Wiesbaden: Springer Fachmedien Wiesbaden GmbH. Zugriff am 16.2.2014. Verfügbar unter: http://nbn-resolving.de/urn:nbn:de:1111-20130707695 
Geertz, C. (1987). Dichte Beschreibung: Beiträge zum Verstehen kultureller Systeme (suhrkamp taschenbuch wissenschaft) (Band 696). Frankfurt am Main: Suhrkamp.

Gehring, P. (2019). Über die Körperkraft von Sprache: Studien zum Sprechakt (1. Auflage.). Frankfurt am Main: Campus.

Groth, S. K. \& Samson, K. (2016). Audio Papers - a manifesto. Seismograf/DMT.

Groth, S. K. \& Samson, K. (2019). The Audio Paper: From Situated Practices to Affective Sound Encounters. Paragrana, 28(1), 188-196. https://doi.org/10.1515/para-2019-0013

Haarmann, A. (2019). Artistic Research. Bielefeld: transcript.

Hegel, G. W. F. (2014). Enzyklopädie der philosophischen Wissenschaften III. Werke 10 (suhrkamp taschenbuch wissenschaft) (7. Aufl., Band 1-20, Band 1). Frankfurt am Main: Suhrkamp.

Heider, F. (2005). Ding und Medium. Herausgegeben und mit einem Vorwort versehen von Dirk Baecker. (D. Baecker, Hrsg.). Berlin: Kadmos.

Henke, S., Mersch, D., Van der Meulen, N., Strässle, T. \& Wiesel, J. (Hrsg.). (2020). Manifest der künstlerischen Forschung: eine Verteidigung gegen ihre Verfechter: versetzt mit "Bildstücken"-Deklination einer Collage von Sabine Hertig (2019) = Manifesto of artistic research: a defense against its acvocates: mixed with "Bildstücke"-a declination of the collage by Sabine Hertig (2019) (Denkt Kunst) (1. Auflage.). Zurich: Diaphanes.

Husserl, E. (2009). Ideen zu einer reinen Phänomenologie und phänomenologischen Philosophie. 1. Buch. Allgemeine Einführung in die reine Phänomenologie (Nachwort (1930) (Philosophische Bibliothek). (E. Ströker, Hrsg.). Hamburg: Felix Meiner Verlag.

Jacob, F. (2000). Die Maus, die Fliege und der Mensch: über die moderne Genforschung (dtv) (Ungekürzte Ausg.). München: Dt. Taschenbuch-Verl.

Kelly, N. (2020). Divesting from people pleasing: A mini series by Nicole Kelly. The Heart. Podcast,. Zugriff am 29.7.2020. Verfügbar unter: https://www. theheartradio.org/divesting-from-people-pleasing

von Kleist, H. (2002). Über die allmähliche Verfertigung der Gedanken beim Reden. Kleist-Archiv Sembdner, Heilbronn. Archive,. Zugriff am 9.7.2013. Verfügbar unter: http://www.kleist.org/texte/UeberdieallmaehlicheVerfertigung derGedankenbeimRedenL.pdf

Klenk, M. (2020). Sprechendes Denken: Essays zu einer experimentellen Kulturwissenschaft (Edition Kulturwissenschaft). Bielefeld: transcript.

Kolesch, D. (2006). Wer sehen will, muss hören: Stimmlichkeit und Visualität in der Gegenwartskunst (Suhrkamp Taschenbuch Wissenschaft). In D. Kolesch \& S. Krämer (Hrsg.), Stimme: Annäherung an ein Phänomen (1. Aufl., S. 40-64). Frankfurt am Main: Suhrkamp. 
König, E. \& Brandt, J. G. (2006). Die Stimme - Charakterisierung aus linguistischer Perspektive (Suhrkamp Taschenbuch Wissenschaft). In D. Kolesch \& S. Krämer (Hrsg.), Stimme: Annäherung an ein Phänomen (1. Aufl., S. 111-129). Frankfurt am Main: Suhrkamp.

Krämer, S. (2001). Sprache, Sprechakt, Kommunikation: sprachtheoretische Positionen des 2o. Jahrhunderts (Suhrkamp Taschenbuch Wissenschaft) (1. Aufl.). Frankfurt am Main: Suhrkamp.

Krämer, S. \& König, E. (Hrsg.). (2002). Gibt es eine Sprache hinter dem Sprechen? (suhrkamp taschenbuch wissenschaft). Frankfurt am Main: Suhrkamp.

Lagaay, A. (2011). Zwischen Lautlichkeit und Stille. Das Spiel von Präsenz und Absenz in der Stimme (Eikones). In M. Butte \& S. Brandt (Hrsg.), Bild und Stimme (S. 101-118). München: Wilhelm Fink.

Makagon, D. \& Neumann, M. (2009). Recording culture: Audio documentary and the ethnographic experience. Los Angeles: SAGE.

Marcus, G. E. (2007). Ethnography two decades after writing culture: From the experimental to the baroque. Anthropological Quarterly, 8o(4), 1127-1145.

Marcus, G. E. \& Cushman, D. (1982). Ethnographies as Texts. Annual Review of Anthropology, 11, 25-69.

Mauss, M. (1989). Die Techniken des Körpers (Fischer Wissenschaft). In E. Moldenhauer, H. Ritter \& A. Schmalfuß (Übers.), Soziologie und Anthropologie 2: Gabentausch. Soziologie und Psychologie. Todesvorstellungen. Körpertechniken. Begriff der Personn (4.-5. Tausend., S. 197-220). Frankfurt am Main: Fischer-Taschenbuch-Verl.

Mcluhan, M. (1994). Understanding media: The extensions of man. Cambridge, Massachusetts; London, England: MIT Press.

Menke, C. (2014). Das Kunstwerk: zwischen Möglichkeit und Unmöglichkeit (suhrkamp taschenbuch wissenschaft). In Die Kraft der Kunst (3. Aufl., S. 1740). Berlin: Suhrkamp.

Mersch, D. (2006). Präsenz und Ethizität der Stimme (Suhrkamp Taschenbuch Wissenschaft). In D. Kolesch \& S. Krämer (Hrsg.), Stimme: Annäherung an ein Phänomen (1. Aufl., S. 211-236). Frankfurt am Main: Suhrkamp.

Mersch, D. (2015). Epistemologien des Ästhetischen (Denkt Kunst) (1. Auflage.). Zürich: Diaphanes.

Miller, J.-A. (2007). Jacques Lacan and the voice (SUNY series in psychoanalysis and culture). In V. Voruz \& B. Wolf (Hrsg.), The later Lacan: An introduction (S. 137-146). Albany: State University of New York Press.

Mittelstraß, J. (1982). Versuch über den Sokratischen Dialog (suhrkamp taschenbuch wissenschaft). In Wissenschaft als Lebensform: Reden über philosophische Orientierungen in Wissenschaft und Universität (1. Aufl., S. 138-161). Frankfurt am Main: Suhrkamp. 
Nancy, J.-L. (2010). Zum Gehör (Transpositionen). (E. von der Osten, Übers.). Zürich: Diaphanes-Verl.

Porombka, S. \& Schulze, H. (2018a). Ein Gespräch über Audiopaper. Berlin: Universität der Künste. Zugriff am 3.10.2018. Verfügbar unter: https: //soundcloud.com/stephanporombka/stephan-porombka-holger-schulzeein-gesprach-uber-audiopaper

Porombka, S. \& Schulze, H. (2018b). »Eine Textur, in der das >Textgerede als Produktionssystem erscheint«. Ein Gespräch über Audio Paper (Szenen / Schnittstellen). In D.-C. Assmann \& N. Menzel (Hrsg.), Textgerede: Interferenzen von Mündlichkeit und Schriftlichkeit in der Gegenwartsliteratur. (S. 267-281). München: Wilhelm Fink Verlag.

Rheinberger, H.-J. (1992). Experiment, Differenz, Schrift: zur Geschichte epistemischer Dinge. Marburg an der Lahn: Basilisken-Press.

Schafer, R. M. (1993). The soundscape: Our sonic environment and the tuning of the world. Rochester, Vt.: Destiny Books.

Schenker, C. (2012). Einsicht und Intensivierung: Überlegungen zur künstlerischen Forschung (Schriftenreihe des Instituts für Gegenwartskünste). In E. Bippus (Hrsg.), Kunst des Forschens: Praxis eines ästhetischen Denkens (2. Aufl., S. 79-89). Gehalten auf der Tagung, Zürich: Diaphanes.

Schroer, M. (2004). Gewalt ohne Gesicht: Zur Notwendigkeit einer umfassenden Gewaltanalyse (Edition Suhrkamp). In W. Heitmeyer \& H.-G. Soeffner (Hrsg.), Gewalt: Entwicklungen, Strukturen, Analyseprobleme (Band 2246 : Kul, S. 151-173). Frankfurt am Main: Suhrkamp.

Schulze, H. (2017). How to think sonically? On the generativity of the flesh (Thinking media). In B. Herzogenrath (Hrsg.), Sonic thinking: A media philosophical approach (S. 217-242). New York: Bloomsbury Academic, an imprint of Bloomsbury Publishing, Inc.

Steiner, U. C. (2012). Ohrenrausch und Götterstimmen: eine Kulturgeschichte des Tinnitus. München: Wilhelm Fink.

Stierle, K. (1996). Gespräch und Diskurs: Ein Versuch im Blick auf Montaigne, Descartes und Pascal (Poetik und Hermeneutik). In K. Stierle \& R. Warning (Hrsg.), Das Gespräch (2., unveränd. Aufl., S. 297-334). München: Fink.

Voegelin, S. (2010). Listening to noise and silence: Towards a philosophy of sound art. New York: Continuum.

Weihrich, M. (2017). Handeln (Handbuch). In R. Gugutzer, G. Klein \& M. Meuser (Hrsg.), Handbuch Körpersoziologie, Band 1: Grundbegriffe und theoretische Perspektiven (Band 1-2, Band 1, S. 33-37). Wiesbaden: Springer VS. 